\title{
Semantic categorization in a linear order problem
}

\author{
REBECCA M. PLISKE and KIRK H. SMITH \\ Bowling Green State University, Bowling Green, Ohio 43403
}

\begin{abstract}
The present investigation was conducted to determine whether subjects could use categorical codes based on semantic memory information (gender of names) to make rapid decisions about the order of names in a linear series. Subjects were taught linear order problems in which 12 names (six male and six female) were either randomly ordered or blocked by sex. The results support a dual-process model which proposes that subjects use both categorical information (discrete linguistic codes) and serial position information when asked to make mental comparisons of arbitrarily ordered items. Furthermore, the data indicate that both the ordinal distance between the terms in the test pair (step size) and the serial position of the test terms in the linear order affect the reaction time to a particular test comparison.
\end{abstract}

Recent studies of mental comparisons have consistently found that, when comparing two items on a particular dimension (e.g., size, height, etc.), the more discrepant the two stimuli, the easier the comparison. This phenomenon is known as the distance effect (Potts, Banks, Kosslyn, Moyer, Riley, \& Smith, 1978). Much of the research concerning the distance effect has involved the retrieval and use of information in semantic memory. For example, digits (Moyer \& Landauer, 1967), animal names (Moyer, 1973), and object names (Paivio, 1975) were used as stimuli in these studies. It is also possible to teach subjects an arbitrarily ordered sequence, test them on comparisons, and still obtain the distance effect. For example, Potts (1972) presented subjects with a paragraph that contained several sentences of the form, "The $\mathrm{X}$ was friendlier than the $\mathrm{Y}$," where $\mathrm{X}$ and $Y$ were animal names. After studying the paragraph, subjects were asked to make pairwise comparisons. As in previous studies, reaction time decreased as the distance between the items on the imposed scale increased.

Many different models have been proposed to account for the distance effect. These models can be grouped into the following categories: analog models, discrete coding models, dual-process models, and serial position models. Each model makes certain assumptions

This report is based on a thesis submitted in partial fulfillment of the requirements for the Master of Arts degree at Bowling Green State University by the first author, and was presented in part at the annual meeting of the Midwestern Psychological Association in Chicago, llinois, May 1978. We gratefully acknowledge the comments on earlier forms of this manuscript made by D. V. DeRosa, B. T. Mynatt, and L. Schipper, the first author's research committee, and by K. J. Holyoak and R. D. Tweney. This research was supported in part by National Science Foundation Grant BNS77-16860 to Bowling Green State University, Kirk H. Smith and Barbee T. Mynatt, coprincipal investigators. Reprint requests should be sent to Kirk H. Smith, Department of Psychology, Bowling Green State University, Bowling Green, Ohio 43403. about the type of representation and the processes involved in the mental comparison task.

Analog models assume that the mental comparison task involves the comparison of images (cf. Paivio, 1975 ) or the comparison of some other type of continuous representation of the stimulus items (cf. Moyer \& Bayer, 1976). According to these models, the distance effect is based on the absolute difference between the analog representations on the scale in question (e.g., size, height, etc.). The larger the absolute difference between the items, the faster the comparison process. It is assumed that subjects are making some type of internal psychophysical judgment analogous to the judg. ments made in perceptual studies (e.g., Johnson, 1939).

Discrete coding models (cf. Banks, 1977) assume that the stimulus items are represented as discrete linguistic codes (e.g., "small," "large"). According to Banks (1977), the distance effect occurs because the probability that two items receive identical codes during the initial encoding stage decreases as the distance between the items increases. If the items receive identical codes, additional discrimination processes are required, which increases reaction time.

Kosslyn, Murphy, Bemesderfer, and Feinstein (1977) recently proposed a dual-process model of comparative judgment that includes both discrete and analog representations of the stimulus items. Kosslyn et al. attempted to test the various models of comparative judgment in the following experiment. They taught subjects the absolute sizes of six stick men that were each a different color. In addition, they trained the subjects to categorize the three larger figures as "large" and the three smaller figures as "small." Subjects learned the sizes, colors, and category labels to a criterion of either $200 \%$ or $500 \%$ overlearning before they were tested on pairs of color names. The results of the Kosslyn et al. study indicate that the amount of overlearning determined whether or not the distance effect occurred. Distance effects oc- 
curred in the $200 \%$ overlearning group for both withincategory comparisons and between-category comparisons; in the $500 \%$ overleaming group, distance effects occurred only for the within-category comparisons. Kosslyn et al. concluded that subjects in the $500 \%$ overlearning group used analog representations when the objects to be compared came from the same category, but that when the objects came from different categories, the category labels (discrete codes) provided sufficient information to make the comparison.

The results of the Kosslyn et al. (1977) study strongly support a dual-process model because they found both the distance effect and a blocking (categorization) effect. The distance effect indicates that subjects were processing the size information, which was assumed to be in analog form, and the blocking effect indicates that subjects were also using discrete linguistic codes when making a mental comparison. Unfortunately, the robustness of the blocking effect demonstrated by Kosslyn et al. is questionable in light of the results of a recent study by Woocher, Glass, and Holyoak (1978).

Woocher et al. (1978) taught subjects two 8-term linear orders which involved occupation names ordered on the dimension of "tallness." After testing subjects on the two separate orders, Woocher et al. told the subjects that all eight people in one order were taller than all eight people in the other order. Thus, it was possible for subjects to hook the two orders together to form a 16-term order. Next Woocher et al. tested their subjects on the 16-term order. They then split their subjects into two groups, one of which received training designed to make the division of the 16-term order into two smaller 8-term orders more salient. The training consisted of pressing one of two buttons as quickly as possible to indicate whether the name belonged to the "tall" or "short" half of the 16-term order. The three repetitions of the 16 terms lasted approximately $15 \mathrm{~min}$. When subjects in the trained group were then retested on pairs that involved one name from the "tall" group and one name from the "short" group, they were expected to respond on the basis of group membership (a categorical decision).

Woocher et al. (1978) found distance effects both for the 8 -term orders and for the 16-term order. Furthermore, they found strong serial position effects even when the ordinal distance between the terms to be compared (i.e., step size) was held constant. The serial position curves for all the step sizes resemble the traditional inverted U-shaped curves found in serial learning studies. These data indicate that the "distance effect" found in the Woocher et al. study may be an artifact produced by the serial position of the terms. Previous linear order studies (e.g., Potts, 1974; Trabasso \& Riley, 1975) have also reported bow-shaped serial position curves. However, these studies did not include a sufficient number of remote comparisons to allow assessment of serial position effects independent of the distance between terms.
Woocher et al. (1978) claim that their data are best explained by the "positional discriminability hypothesis" (Crowder, 1976), which has been proposed to account for the serial position effect in traditional serial learning. This hypothesis assumes that ordered stimuli are mapped onto some type of internal array. The central positions of the internal array are less discriminable than the end position. The positional discriminability hypothesis can account for the distance effect, since as the distance between items on an imposed scale increases, the items necessarily become closer to the end terms of the order. Strong support for the positional discriminability model comes from a multiple-regression analysis on the reaction time data collected in the 16-term order problem in the Woocher et al. (1978) study. The "distance from end anchor" variables accounted for $45 \%$ of the total variance. These variables were minimal distance from an end term and minimal distance from the "tallest" end term. The distance between the two terms in the test pair accounted for a small, but significant, amount of residual variance. Surprisingly, the factor of within-block vs. between-block comparisons was not significant after the variance due to distance and end anchors was removed.

The results of the Woocher et al. (1978) study are problematic for all three models discussed above. Their data suggest that the "distance" effect is not due to the difference between analog representations, but rather that it is simply an artifact of the serial position of the terms in the order. Furthermore, the lack of a blocking effect due to the category labels suggests that subjects (even those who were explicitly trained on the category labels) were unable to use this discrete linguistic information to facilitate the comparison process.

It is possible that Woocher et al. (1978) failed to obtain a blocking effect due to the training procedure they used. The Kosslyn et al. (1977) study indicates that category labels must be highly overlearned in order to facilitate mental comparisons. The subjects in the Woocher et al. study first learned two separate 8-term orders and were subsequently told that the two orders could be combined. Perhaps the blocking manipulation would have been more effective if it had been introduced during the initial learning of the linear order and if the different categories were made more salient by using highly overlearned categories (e.g., sex).

In order to investigate this possibility, the present study used linear order problems which consisted of 12 names (six male and six female) ranked in order of intelligence. One group of subjects (random group) received a problem in which the 12 names were randomly ordered. The other group of subjects (blocked group) received a problem in which the names were blocked by sex, so that either all six males were smarter than all six females, or vice versa. Since the blocking manipulation was instigated during the initial part of the experiment, it was predicted that subjects in the 
blocked group would be able to utilize the sex blocking to facilitate the between-block comparisons.

\section{METHOD}

\section{Subjects}

The subjects were 28 undergraduate students. Fourteen students were randomly assigned to each of the two conditions. Subjects received credit toward a course requirement for their participation in the experiment. The data from six subjects (three from each condition) were not included in the analyses because their error rate exceeded $10 \%$.

\section{Materials}

A 12-term linear order was constructed from names chosen from Battig and Montague (1969) category norms with the following restrictions: (a) No two names started with the same letter, (b) all names were one syllable in length, and (c) all names were four letters long. The order consisted of six male and six female names. For the random group, the names were randomly ordered with the restriction that no more than three names of the same sex could be grouped together. For the blocked group, however, the names were blocked by sex.

\section{Procedure}

The procedure was identical for both groups of subjects. When subjects signed up for the experiment, they received a list of 12 names which they were instructed to memorize (in the correct order) before coming to the experimental session. At the beginning of the experimental session, subjects were given a recall task in which they were required to write down the names on the list in serial order. All of the subjects were able to do this.

After the recall task, subjects were seated at the typewriter console of a Digivue plasma display screen under the control of a NOVA 1220 computer. Pairs of names were presented on the display screen, and subjects responded by pressing one of two typewriter keys. The computer recorded the reaction time and the response for each comparison. Two keys on the typewriter console (one on the left and one on the right) were designated by arrows pointing to them. Subjects were instructed to push the button under the smarter person. They were also instructed to work as quickly as possible, without sacrificing accuracy.

Subjects went through 20 practice trials which involved pushing the button under the larger of two numbers presented on the screen in front of them. After completing the practice trials, subjects were given another recall task to insure the practice trials had not interfered with the linear order they had previously learned. Again, all subjects were able to perform the recall task.

After completing the second recall task, subjects were presented with 132 test comparisons. These comparisons included the 66 possible combinations of the 12 terms in the linear order problem. Each comparison was shown in both forward order (e.g., A-B) and reverse order (e.g., B-A). The test comparison remained on the screen until the subject responded. Feedback (i.e., "correct" or "wrong") appeared for $1,500 \mathrm{msec}$ following the subject's response. The screen then went blank for $500 \mathrm{msec}$ before the next test comparison appeared. Each subject received a different random presentation order of the 132 test com?arisons.

\section{RESULTS}

An analysis of variance revealed that the main effect lue to the order of the presentation of the pairs on the icreen (forward vs. reverse) was not significant, although $t$ approached significance $[\mathrm{F}(1,20)=3.22, \mathrm{MSe}=.322]$. Furthermore, presentation order did not interact signifi- cantly with any of the other factors. Since there were no reliable differences between the order of presentation of the test pairs, all subsequent analyses were based on mean reaction times averaged over presentation order.

The overall error rate was fairly low $(5.4 \%$ in the blocked group and $4.8 \%$ in the random group). When subjects made an incorrect response to only one of the presentation orders of a particular test comparison, their reaction time for that comparison was based on their correct response. On occasion a subject responded incorrectly to both presentation orders of a particular test comparison. When this occurred, which was very rarely, the mean reaction time of the entire group for that comparison was substituted. Therefore, as in previous mental comparison studies, subsequent analyses were based solely on correct responses.

In Figure 1, mean correct reaction time is plotted as a function of step size ${ }^{1}$ and type of comparison (withinblock vs. between-block). The functions for the withinblock comparisons were nearly identical for both groups. That is, reaction time decreased as step size increased for both the random and blocked groups $[F(1,20)=$ $84.36, \mathrm{MSe}=.075$, by a linear trend test $]$. There was no significant interaction with groups. These results indicate that distance effects occurred with the within-block comparisons for both the random and blocked groups.

Although the functions for the within-block comparisons were very similar for both groups of subjects, the functions for the between-block comparison were quite different for the two groups. An orthogonal polynominal trend analysis indicated a significant linear trend that interacted with groups $[\mathrm{F}(1,20)=29.06$, $\mathrm{MSe}=.427 \mathrm{~J}$. The results of these analyses support what the reader can see in Figure 1. That is, distance effects occurred for the between-block comparisons for the random group but not for the blocked group.

The data presented in Figure 1 also indicate that there was an interaction between type of comparison (between-block vs. within-block) and groups. Betweenblock comparisons $(.98 \mathrm{sec})$ were faster than within-

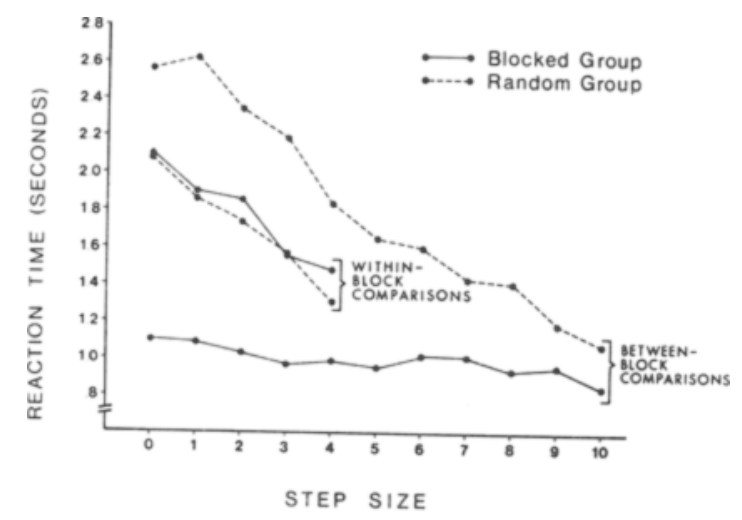

Figure 1. Mean reaction time as a function of step size and type of comparison. Note that identical comparisons were tested in both the random and blocked groups, so that the "betweenblock" comparisons for the random group do not involve a categorized ordering. 
block comparisons $(1.78 \mathrm{sec})$ for the blocked group, whereas between-block comparisons $(1.80 \mathrm{sec})$ were slower than within-block comparisons $(1.71 \mathrm{sec})$ for the random group $[F(1,20)=51.43$, MSe $=.416]$. This indicates that subjects in the blocked group were able to utilize the sex blocking to facilitate the comparison process. Furthermore, there was no effect of distance on the between-block comparisons for the blocked group. It appears that when subjects in the blocked group were presented with an opposite-sex pair, they were able to make a categorical decision on the basis of the sex of the terms, and this decision was not affected by the distance between the terms.

To examine the data for serial position effects, reaction time was plotted as a function of the smarter term in the comparison. The serial position curves for the adjacent comparisons (i.e., Step Size 0) for both the random and blocked groups are shown in Figure 2. Although the data for the random group are somewhat noisy, they show the traditional inverted U-shaped function found in serial learning experiments. In contrast, the serial position curve for the blocked group shows a deep drop in the middle. This offers further support for the claim that subjects in the blocked group were able to utilize the sex blocking. The midpoints of the serial position curve shown in Figure 2 actually represent the end points for the two smaller 6-term orders that the subjects in the blocked group were able to form due to the sex blocking.

Reaction time data from the other step sizes were also plotted as a function of the serial position of the smarter term. Because a single figure presenting curves for all the step sizes is uninterpretable, Figures 3 and 4 show the serial position curves for selected step sizes (i.e., 1, 3, 5, and 7) for the random and blocked groups, respectively. Figure 3 shows that when ordinal distance between the terms (i.e., step size) was held constant, there were still strong effects of serial position for the

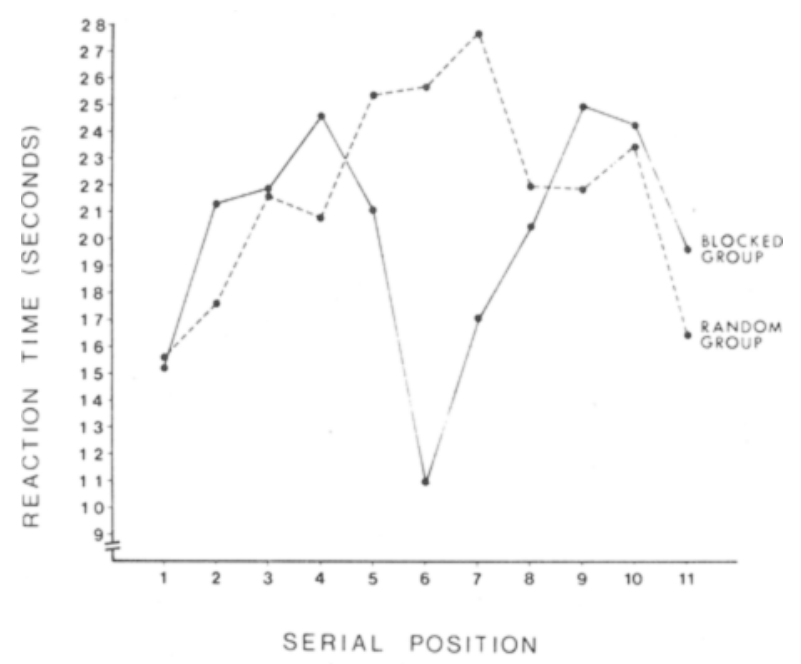

Figure 2. Mean reaction time to adjacent comparisons as a function of serial position of the smarter term.

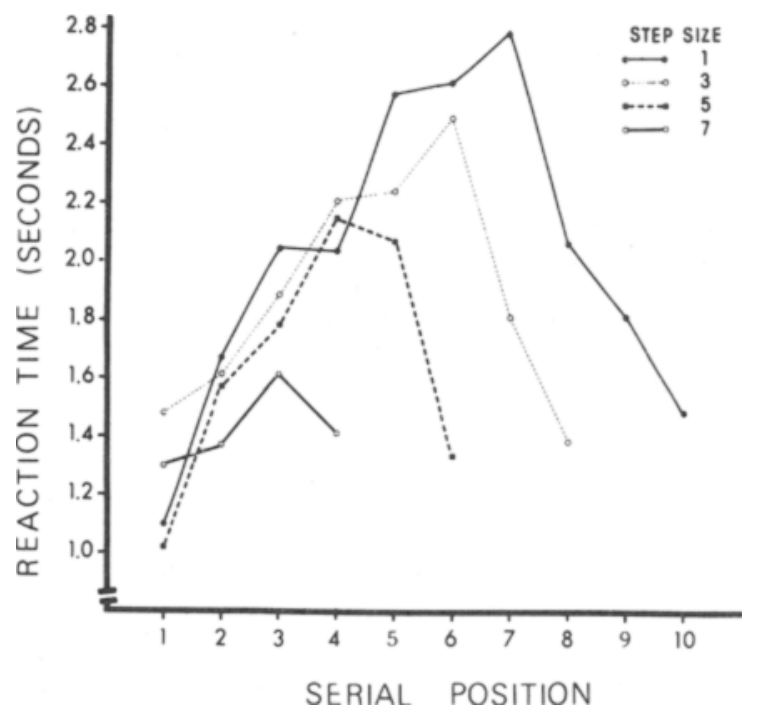

Figure 3. Mean reaction time to nonadjacent comparisons as a function of serial position of the smarter term-random group.

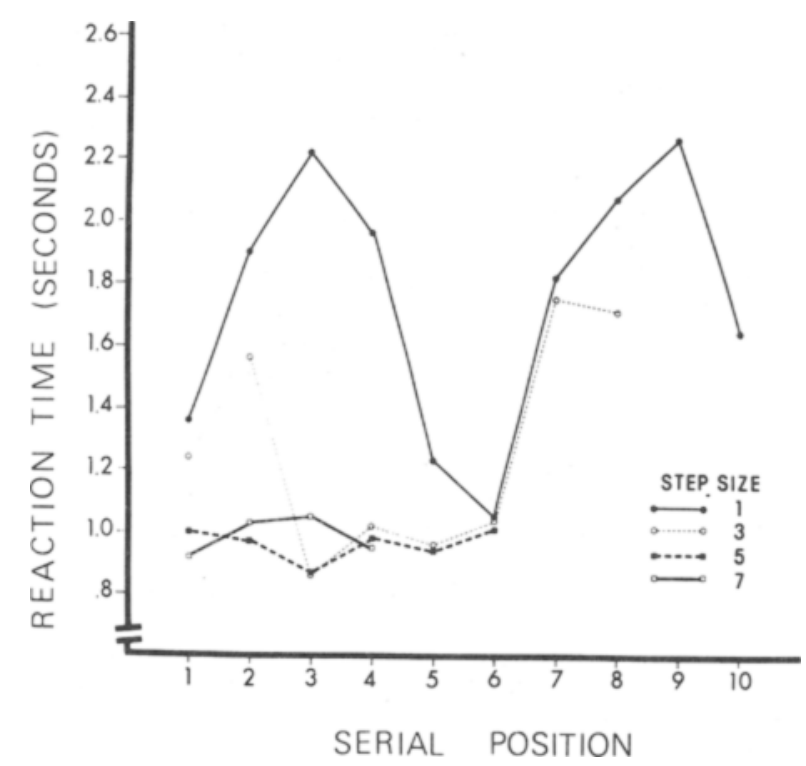

Figure 4. Mean reaction time to nonadjacent comparisons as a function of serial position of the smarter term-blocked group.

random group. However, the fact that the serial position curve for Step Size 1 is higher than the curve for Step Size 3, which is in turn higher than the curves for Step Sizes 5 and 7, indicates that there was still an effect of distance when serial position of the "smarter" term was held constant. ${ }^{2}$

Referring to Figure 4, the reader will note that when the ordinal distance between the terms was held constant, reaction time for the blocked group also showed strong serial position effects. The serial position curves for the smaller step sizes ( 1 and 3 ) for the blocked group show the large dip at the middle points of the order. The longer step sizes (5 and 7) involve only between-block comparisons. Therefore, the serial position curves for these step sizes are relatively flat. This, 
once again, indicates that the sex blocking was effective.

To insure that the distance effects obtained in the present investigation were not due solely to the end terms, the data were reanalyzed excluding all pairs involving either of the end terms. The reaction time data (excluding end terms) for both groups of subjects are plotted in Figure 5. Once again, a significant linear trend for the mean reaction times for the within-block comparisons was found $[\mathrm{F}(1,20)=68.26, \mathrm{MSe}=.055]$. This trend did not interact with groups $[F(1,20)=$ $.085, \mathrm{MSe}=.055]$. An orthogonal polynomial trend analysis found a reliable linear trend for the mean reaction times for the between-block comparisons $[F(1,20)=22.01, \mathrm{MSe}=.301]$, which interacted sig. nificantly with groups $[\mathrm{F}(1,20)=19.94, \mathrm{MSe}=.301]$. Thus, although the data are somewhat noisier when the comparisons involving the end terms are removed, the differences shown in Figure 5 are still statistically reliable.

\section{DISCUSSION}

The results of the present investigation appear to be best explained by a dual-process model of comparative judgment. The fact that we found clear effects due to the blocking manipulation indicates that subjects can (and do) use discrete information when making mental comparisons. The results also indicate that both the serial position of the terms in the linear order and the distance between the terms affect the reaction time for a particular comparison. This suggests that some type of internal memory scanning process is also involved in the processing of artificial order problems. The question is how best to characterize the combined operation of these two processes.

We propose that subjects in linear order studies form some type of internal representation of the order that preserves the serial order of the terms. This representation could be an analog (or visual) representation of the terms as they would appear on an external list (i.e., $\mathrm{A}>\mathrm{B}>\mathrm{C}>\mathrm{D}>\mathrm{E}$ ). Alternatively, the representation

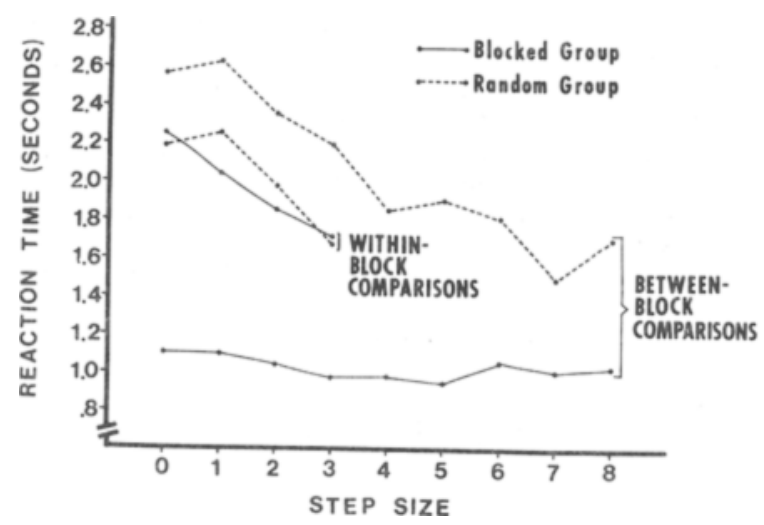

Figure 5. Mean reaction time as a function of step size with comparisons involving end terms excluded. could be strictly propositional (or abstract) in nature. All that is essential about the mental representation is that it preserves the serial order of the terms in some type of internal array.

In addition to storing an internalized representation of the linear order, it is assumed that subjects have also stored additional information about the terms in the form of discrete facts. For subjects in the blocked group, the sex of the name would be part of the representation, along with a decision rule such as: All males are smarter than all females. Presented with the pair ANNE-MIKE, the subjects in the blocked group would be able to process the fact that this was a female-male pair. A rapid response could then be made to the button under the male name. When subjects in the blocked group received the pair MIKE-PAUL, they would process the fact that that was a same-sex pair. Failure of the decision rule would indicate further processing was necessary before a response could be made.

When there is no categorical information to facilitate the comparison process, the subject must search his/her internal array before responding. The exact nature of this search cannot be determined from the data collected in the present investigation. However, the inverted U-shaped curve found for the random group seems to support Woocher's (1976) explanation that subjects search in parallel from the two end terms of the 12-term order. Similarly, the two smaller inverted U-shaped curves found for the blocked group suggest that these subjects may have searched in parallel from the two end terms of the appropriate sex block.

The assumption that subjects store more than one type of information about the linear order in their memories is consistent with a recent investigation by Lawson (1977), who demonstrated that subjects in a linear order task store propositional information, as well as order information, about the terms in the linear order. Lawson used a sentence classification task to assess memory for sentences presented during the learning phase of a 7-term linear order task. His results indicate that subjects are able to discriminate true implications from previously presented sentences and their paraphrases. Thus, subjects must store some type of information about which two terms were presented together during the learning phase. Lawson suggested that the sentences are represented in the form of propositions such as (higher, A, B). In a subsequent reaction time task, Lawson found no overall reaction time differences between types of sentences (olds, paraphrases, and implications). However, he did find ordinal distance effects for sentences that did not involve end terms. Thus, Lawson concluded that subjects have both propositional and ordinal information about the terms in a linear order stored in memory.

The dual-process model outlined here is similar to the Kosslyn et al. (1977) model discussed in the introduction. However, the Kosslyn et al. model was pro- 
posed to account for the mental comparison processes involved in the comparison of objects that are stored in memory in terms of absolute size, but not as an ordered sequence. Therefore, the Kosslyn et al. model includes an image comparison process in which the absolute sizes of the items can be compared in a manner analogous to actual perceptual comparisons. The subjects in the present investigation were never presented with absolute measures of the different people's intelligence. Thus, they could store only ordinal information about the terms in the linear order. Therefore, the second process proposed here is not the analog comparison process proposed by Kosslyn et al., but rather some type of internal scanning process.

It appears that in order for category information to have a facilitating effect on mental comparisons, the categories must either be based on semantic memory information (e.g., the use of sex blocking in the present study) or they must be highly overlearned (e.g., the use of the $500 \%$ overlearning criterion by Kossiyn et al., 1977). It is important to note that there is a difference between the blocking used in the Kosslyn et al. and Woocher et al. studies and the blocking used in the present investigation. Kosslyn et al. and Woocher et al. had to teach subjects the category labels. In contrast, in the present investigation no explicit instructions about the sex blocking were given. Subjects in the present investigation knew that PAUL was a male name before they came to the experiment. When subjects in the blocked group received an opposite-sex pair, they could automatically respond on the basis of a decision rule. However, in the Kosslyn et al. and Woocher et al. studies, subjects had to associate the correct category label with the terms to be compared before they could make a decision based on category information. It is possible that there are differences between how subjects process "natural" (or semantic memory) information and how they process "artificial" information. Further investigations should attempt to determine the boundary conditions for the blocking effects found in the present study.

\section{REFERENCES}

BANKs, W. P. Encoding and processing of symbolic information in comparative judgments. In G. H. Bower (Ed.), The psychology of learning and motivation (Vol. 11). New York: Academic Press, 1977. Pp. 101-159.

Battig, W. F., \& Montague, W. E. Category norms for verbal items in 56 categories: A replication and extension of the Connecticut category norms. Journal of Experimental Psychology Monograph, 1969, 80, 1-45.

Crowder, R. G. Principles of learning and memory. Hillsdale, N.J: Erlbaum, 1976.

Johnson, D. M. Confidence and speed in two-category judgment. Archives of Psychology, 1939, No. 241, 1-52.
Kosslyn, S. M., Murphy, G. L., Bemesderfer, M. E., \& Feinste in, K. J. Category and continuum in mental comparisons. Journal of Experimental Psychology: General, 1977, 106, 341-375.

LAwson, R. Representation of individual sentences and holistic ideas. Journal of Experimental Psychology: Human Learning and Memory, 1977, 3, 1-9.

MOYEr, R. S. Comparing objects in memory: Evidence suggesting an internal psychophysics. Perception \& Psychophysics, 1973, 13, 180-184.

MOYER, R. S., \& BAYER, R. H. Mental comparison and the symbolic distance effect. Cognitive Psychology, 1976, 8, 228-246.

Moyer, R. S., \& Landauen, T. K. The time required for judgments of numerical inequality. Nature, 1967, 215, 1519-1520.

Paivio, A. Perceptual comparisons through the mind's eye. Memory \& Cognition, 1975, 3, 635-647.

PotTs, G. R. Information processing strategies used in the encoding of linear orderings. Journal of Verbal Learning and Verbal Behavior, 1972, 11, 727-740.

Potrs, G. R. Storing and retrieving information about ordered relationships. Journal of Experimental Psychology, 1974, 103, 431-439.

Potts, G. R., Banks, W. P., Kosslyn, S. M., Moyer, R. S., Riley, C. A., \& Smith, K. H. Encoding and retrieval in comparative judgments. In N. J. Castellan \& F. Restle (Eds.), Cognitive theory (Vol. 3). Hillsdale, N.J: Erlbaum, 1978. Pp. 241-308.

Trabasso, T., \& Riley, C. A. On the construction and use of representations involving linear order. In R. L. Solso (Ed.), Information processing and cognition: The Loyola symposium. Hillsdale, N.J: Erlbaum, 1975. Pp. 381-410.

Trabasso, T., Riley, C. A., \& Wilson, E. G. The representation of linear order and spatial strategies in reasoning: A developmental study. In R. Falmagne (Ed.), Psychological studies of logic and its development. Hillsdale, N.J: Erlbaum, 1975.

Woocher, F. D. Storage and retrieval of information from artificial linear orders. Unpublished doctoral dissertation, Stanford University, 1976.

Woocher, F. D., Glass, A. L., \& Holyoak, K. J. Positional discriminability in linear orders. Memory \& Cognition, 1978, 6, 165-173.

\section{NOTES}

1. Following Trabasso, Riley, and Wilson (1975), the distance between the terms in a test pair will be discussed with respect to step size. Step size is the number of terms in between the two terms that are being compared.

2. A multiple-regression analysis of the data from the random group indicated that the distance (step size) factor accounted for a small but significant amount of variance after the variance due to the serial position of the test terms was removed. The serial position of a test pair is difficult to define, since there are actually two serial positions involved in each test comparison. In this analysis, the serial position of the "smarter" term, the serial position of the "dumber" term, and the minimal distance of either term from an end term were used as "serial position variables." The serial position of the "smarter" term and the minimal distance from an end term both accounted for significant amounts of variance, while the serial position of the "dumber" term was not a significant predictor of RT.

(Accepted for publication May 9, 1979.) 